\title{
La complejidad del mercado bursátil latinoamericano a partir de un modelo autómata celular conductual
}

\author{
The Complexity of Latin-American Stock Market \\ using a Behavioral Cellular \\ Automaton Model
}

Complexidade do mercado de ações da America Latina através de um modelo autômato celular comportamental

Leonardo Hernán Talero-Sarmiento * Juan Benjamín Duarte-Duarte ** Laura Daniela Garcés-Carreño ***

DOI: https://doi.org/10.19053/01203053.v36.n64.2017.5421

Fecha de recepción: 18 de septiembre de 2016

Fecha de aceptación: 25 de mayo de 2017

\footnotetext{
* Ingeniero Industrial. Estudiante de Maestría en Ingeniería Industrial. Universidad Industrial de Santander. Bucaramanga, Colombia. Correo electrónico: leonardo.talero@gmail.com iD http://orcid.org/0000-0002-4129-9163 Dirección: Carrera 27 Calle 9, Ciudad Universitaria, Edif. Escuela de Estudios Industriales y Empresariales, Of. 207-25, Universidad Industrial de Santander. Bucaramanga, Colombia.

** Ingeniero Industrial. Doctor en Finanzas de Empresa. Profesor titular. Escuela de Estudios Industriales y Empresariales. Universidad Industrial de Santander. Bucaramanga, Colombia jduarte@uis.edu.co iD http://orcid. org/0000-0002-3630-7683

***Ingeniera Industrial. Estudiante de Maestría en Ingeniería Industrial. Profesor auxiliar. Escuela de Estudios Industriales y Empresariales. Universidad Industrial de Santander. Bucaramanga, Colombia. lauradgarces@gmail. com (D) http://orcid.org/0000-0002-3630-7683
} 


\section{Resumen}

La presente investigación busca evaluar el nivel de complejidad del mercado latinoamericano, mediante la construcción de un modelo autómata celular. Para ello se estudian seis índices bursátiles: COLCAP, IPSA, MERVAL, MEXBOL, SPBLPGPT e IBOV, en el periodo 2004-2016. Estas series son analizadas a partir de su comportamiento estadístico, el ajuste de retornos y la estimación de su grado de complejidad. Este último es contrastado posteriormente con el nivel de complejidad obtenido mediante la simulación de un mercado bursátil artificial, y se concluye que los mercados latinoamericanos, a pesar de presentar diferencias, suelen tener tendencias similares, ya que su grado de complejidad no puede ser pronosticado por un modelo autómata celular conductual basado netamente en la imitación.

Palabras clave: finanzas conductuales, principios subyacentes, técnicas computacionales, modelado y simulación.

JEL: C63, G02. 
Apuntes CENES Volumen 36, Número 64

julio - diciembre 2017. Págs. 199-223

\section{Abstract}

The aim of this research is to evaluate the complexity level of Latin-American stock market using a cellular automaton model. For this purpose six indexes are studied: COLCAP, IPSA, MERVAL, MEXBOL, SPBLPGPT and IBOV respectively, during the period 2004 and 2016. The series are analyzed from their statistical behavior, adjustment of returns and estimation of its complexity. The last one is contrasted with the complexity level obtained simulating an artificial stock market model. Concluding that although Latin-American stock markets present differences they have similar tendencies and their complexity level cannot be predicted by a purely behavioral cellular automaton model.

Keywords: behavioral finance, underlying principles, computational techniques, simulation modeling. 


\section{Resumo}

Esta pesquisa visa avaliar o nível de complexidade do mercado da América Latina através da construção de um modelo de autômato celular; para isto e feito o estúdio de seis series de ações; COLCAP, IPSA, MERVAL, MEXBOL , SPBLPGPT e IBOV entre 2004-2016: Estas séries são analisados a partir do seu comportamento estatístico, o ajuste de retornos e estimativa da sua complexidade. Este último é então contrastado com o nível de complexidade obtida através da simulação de um mercado de ações artificial, concluindo que os mercados da América Latina, apesar de ter diferenças, muitas vezes têm tendências semelhantes e que a sua complexidade não pode ser prevista por um modelo de autômato celular puramente comportamental.

Palavras-chave: finanças comportamentais; princípios fundamentais; técnicas computacionais; modelagem e simulação. 
Apuntes CENES Volumen 36, Número 64

julio - diciembre 2017. Págs. 199-223

\section{INTRODUCCIÓN}

La creciente importancia económica de los mercados financieros en el mundo se evidencia en el significativo número de estudios que pretenden conocer, describir y, de ser posible, predecir su comportamiento desde diferentes perspectivas, que incluyen la teoría de mercados eficientes enunciada por Fama (1970), según la cual los inversores se comportan racionalmente y los precios de mercado de un activo reflejan toda la información disponible. También está la hipótesis de mercados adaptativos propuesta por Andrew Lo (2005), que concilia la eficiencia del mercado con la teoría conductista, aplicando los principios de la evolución (competencia, adaptación y selección natural). Y está el enfoque conductual defendido por Robert
Shiller (2003), quien manifiesta que los inversores no siempre son racionales y toman sus decisiones impulsados por aspectos inherentes a su naturaleza humana, que incluyen la psicología y la sociología. A partir de dichos estudios se han encontrado diversos comportamientos en los retornos de los activos que no logran ser explicados por modelos lineales, lo cual ha dado fuerza a otra visión del mercado bursátil, que afirma que el caos puede ser derivado de la compleja estructura que conforma un mercado, a raíz de eso, múltiples disciplinas han retomado la hipótesis de mercado fractal descrita por Benoit Mandelbrot (1972).

Teniendo en cuenta las anteriores hipótesis y la facilidad que tiene el coeficiente de Hurst para estimar el comportamiento caótico de las series 
financieras (Sierra, 2007), diversos autores han investigado el grado de complejidad y, de paso, evaluado el nivel de eficiencia de mercados reales y simulados, para así determinar los factores que influyen en la estabilidad o volatilidad bursátil. Algunos de los primeros autores en tratar el tema fueron Wei Ying, Fan y Wang (2003), al construir un mercado virtual en el que se analizó el consenso de sus agentes entre comprar, retener y vender, modelo que evolucionaría mediante la estimación de la eficiencia (Fan, Ying, Wang \& Wei, 2009) y el análisis de la misma, cuando existen políticas económicas nacionales (Ying \& Fan, 2014).

Otros autores, como Bartolozzi y Thomas (2005), Bakker, Hare, Khosravi y Ramadanovic (2010), y Stefan y Atman (2015), evaluaron la complejidad de los mercados a partir de redes de confianza entre los agentes modelados y la información que estos adquirían y generaban. De manera similar, algunos investigadores -como Zhou, Zhou, Wang, Tang y Liu (2004) y Mozafari y Alizadeh (2013)- han propuesto modelos autómatas celulares de aprendizaje, que buscaban establecer reglas de decisión que hagan más dinámico el mercado. De manera paralela, Qiu, Kandhai y Sloot (2007) y Atman y Gonçalves (2012) formularon perfiles de inversores virtuales, con el fin de determinar la complejidad que cada tipo de agente puede producir a un mercado.
La estructura del presente artículo tiene como fin analizar las semejanzas entre las principales series bursátiles latinoamericanas y evaluar si un modelo autómata celular de tipo conductual, como el formulado por Fan (2009), puede simular el grado de complejidad de Latinoamérica; para ello, en el primer capítulo se expone la metodología desde dos perspectivas: una descriptiva, asociada a los datos históricos reales y otra enfocada en la construcción de un mercado simulado. En el segundo capítulo se lleva a cabo un análisis de los principales resultados estadísticos y del comportamiento de las series a lo largo del tiempo. En el tercer capítulo se discuten las teorías y dificultades que se evidenciaron durante la investigación, y en el cuarto se presentan las conclusiones.

\section{METODOLOGÍA}

El comportamiento económico latinoamericano puede ser representado por la dinámica de sus indicadores bursátiles, puesto que dichos índices reflejan el cambio en la economía de cada país al recopilar los precios de cierre de las principales acciones comercializadas en la región. Para esta investigación se analizaron las series COLCAP (Colombia), IPSA (Chile), MERVAL (Argentina), MEXBOL (México), SPBLPGPT (Perú) e IBOV (Brasil) para el periodo 2004-2016, desde dos perspectivas: una real y otra simulada. 
En la primera perspectiva se evalúan semejanzas entre las series históricas bursátiles mediante un estudio empírico, el cual incluye el análisis descriptivo de los índices durante el periodo de estudio. Para ello, se comparan de manera estadística las series financieras a partir de la similitud de sus datos, utilizando estadísticos descriptivos por serie, junto con la correlación (ecuación 1) entre estas.

$$
\rho_{x_{i}, x_{j}}=\frac{\operatorname{COV}\left(x_{i}, x_{j}\right)}{\sigma_{x_{i}} * \sigma_{x_{j}}}
$$

Donde y $x_{j}$ representan dos series financieras diferentes, $\mathrm{COV}$ la covarianza entre estas y $\sigma_{x}$ la desviación estándar de las mismas. En segunda instancia se analizan los cambios históricos de cada índice internacional a partir del ajuste de distribución de sus rentabilidades, utilizando el complemento para análisis de riesgo @RISK de la suite Palisade Corporation en su versión 7.5.0.

Una vez ajustado el comportamiento probabilístico de cada índice, se realiza el estudio de rentabilidades desde dos visiones: el periodo total de la serie comprendido entre 2004-2016 y el análisis de la serie a partir de subperiodos anuales. Finalmente y con el objetivo de inferir el nivel de eficiencia de los mercados bursátiles latinoamericanos, se calcula el nivel de memoria en estos a partir del reescalamien- to estadístico de sus datos año a año, mediante el coeficiente de Hurst. Para la estimación del mismo se construye una función $F\left(x_{i, a}\right)$ (función de reescalamiento, ver ecuación 4) para cada serie bursátil, la cual es definida como la razón entre su desviación estándar (ver ecuación 2) y el rango de sus datos (ver ecuación 3):

$$
\begin{gathered}
\sigma_{x_{i}}=\sqrt{\frac{1}{N} \sum_{t=1}^{N}\left(X_{i, a}-\overline{x_{i, a}}\right)^{2}} \\
R\left(X_{i, a}\right)=\left(\max _{1 \leq i \leq N} X_{(i, a)}-\underset{1 \leq i \leq N}{\left.\min X_{(i, a)}\right)}\right.
\end{gathered}
$$

Donde $x_{i a}$ indica la serie histórica de cada índice, suavizada mediante un promedio móvil de anchura $a$, denominado subperiodo.

$$
F\left(x_{i, a}\right)=\frac{\sigma_{x_{i}}}{R\left(X_{i, a}\right)}
$$

En múltiples sistemas se determina que la función es proporcional a la raíz Hurstiana de T (Fan et al., 2009) (ver ecuación 5). $F\left(x_{i, a}\right)$ por tanto es suavizada logarítmicamente con el fin de obtener el coeficiente de Hurst $(H)$.

$$
F\left(x_{i, a}\right) \propto a * T^{H}
$$

El valor del coeficiente representa el grado de complejidad de una serie; $H=0.5$, cuando, se considera que esta 
es completamente aleatoria tipo ruido blanco. Dicho comportamiento, para el caso de los índices bursátiles, se relaciona con la hipótesis de mercados eficientes (Malkiel \& Fama, 1970). Si el valor de Hurst está comprendi-do entre $\left[\begin{array}{lll}0.5 & y & 1\end{array}\right]$ la serie presenta un comportamiento persistente (Blasco de Las Heras \& Santamaría, 1994), lo que indica una tendencia reiterativa en el mercado, es decir, si un índice o acción va al alza en su precio, lo más probable es que repita esa tendencia (y viceversa). Por otra parte, si el coeficiente es inferior a $0,5[0 \leq H<0.5]$ la serie presenta un comportamiento antipersistente, lo cual indica que si en un momento determinado el índice o una acción va al alza en su precio, lo más probable es que en el siguiente instante presente un comportamiento a la baja (y viceversa).

En la segunda perspectiva (simulación) se construye un mercado bursátil artificial basado en los trabajos de Fan (2009; 2003; 2014), con el cual se busca estimar la complejidad de una bolsa compuesta por agentes conductuales, quienes determinan su posición (comprar, retener o vender) a partir de la tendencia del mercado y la capacidad de imitar a su vecindad.

El mercado bursátil artificial es diseñado mediante un modelo autómata celular (MAC), en el que interactúan agentes que se encuentran ubicados en un eje de coordenadas $(x, y)$. La posi- ción de cualquier agente al comienzo de cada iteración se distribuye de manera uniforme entre comprar, retener $y$ vender, y dicha posición puede ser afectada por su vecindad, teniendo en cuenta que cada $A_{x, y}$ (Agente en la posición $\mathrm{x}, \mathrm{y})$ está rodeado de 8 vecinos (cada uno con una posición inicial aleatoria igualmente distribuida), por tanto siempre va a existir una mayoría en la vecindad de cada $A_{x, y^{*}}$

Para que cada $A_{x, y}$ pueda ser afectado por su vecindad, se le asigna una probabilidad de imitación $P$, la cual varía entre $[0,1]$. Si el valor de $P$ oscila en los cuartiles inferiores $[0<P<0.5]$ el agente $A_{x, y}$ presentará una tendencia a llevar la contraria a su vecindad. Si el valor de $\mathrm{P}$ se encuentra en el rango $[0.5<P<1]$, el agente $A_{x, y}$ se verá influenciado por sus vecinos, finalmente, si $P=0.5$ el agente ignorará la influencia de sus aledaños y mantendrá su posición original.

Además de la disposición de la vecindad y la capacidad de imitación, cada agente $A_{x, y}$ podrá ser influenciado por una variable denominada macrofactor (Mf), la cual representa la tendencia de la economía y se considera al alza si $M f \geq 0.5$ y a la baja en caso contrario. El macrofactor puede alcanzar valores en el rango $[0,1]$, cuando el mercado se encuentra al alza la posición de cada agente $A_{x, y}$ es más susceptible de cambiar hacia comprar y caso contrario (retener) cuando el mercado se 
encuentra a la baja. Dicha influencia se hace más fuerte entre más cerca se encuentre $M f$ de los valores extremos.

La dinámica del cambio de posición y su consenso (cantidad total de agentes comprando, reteniendo y vendiendo al final del día o iteración) influyen en la complejidad del mercado simulado.
Lo anterior se mide a partir de la fractalidad de dicho consenso. En la Tabla 1 se evidencia el modelo de transferencia e imitación propuesto por Fan (Fan et al., 2009) y utilizado en esta investigación, con el cual se puede determinar un nivel de complejidad de un mercado bursátil artificial mediante la estimación del coeficiente de Hurst.

Tabla 1. Matriz de transferencia de posiciones

\begin{tabular}{|c|c|c|c|c|}
\hline Macrofactores & Posición vecindad & \multicolumn{3}{|c|}{ Transferencia de probabilidades } \\
\hline & & Comprar & Retener & Vender \\
\hline \multirow{3}{*}{$\begin{array}{l}\text { Información } \\
\text { positiva }\end{array}$} & Comprar & $(P+M f)$ & $(1-P-M f)^{*} 0,5$ & $(1-\mathrm{P}-\mathrm{Mf}) * 0,5$ \\
\hline & Retener & $(1-P)^{*}\left(0,5+0,5^{*} \mathrm{Mf}\right)$ & $\mathrm{P}$ & $(1-\mathrm{P})^{*}\left(0,5-0,5^{*} \mathrm{Mf}\right)$ \\
\hline & Vender & $(1-P)^{*}\left(0,5+0,5^{*} \mathrm{Mf}\right)$ & $(1-P)^{*}\left(0,5-0,5^{*} \mathrm{Mf}\right)$ & $P$ \\
\hline \multirow{3}{*}{$\begin{array}{c}\text { Información } \\
\text { negativa }\end{array}$} & Comprar & $P$ & $(1-P)^{*}\left(0,5-0,5^{*} \mathrm{Mf}\right)$ & $(1-P)^{*}\left(0,5+0,5^{*} \mathrm{Mf}\right)$ \\
\hline & Retener & $(1-P)^{*}\left(0,5-0,5^{*} \mathrm{Mf}\right)$ & $\mathrm{P}$ & $(1-P)^{*}\left(0,5+0,5^{*} \mathrm{Mf}\right)$ \\
\hline & Vender & $(1-P-M f)^{*} 0,5$ & $(1-P-M f) * 0,5$ & $(\mathrm{P}+\mathrm{Mf})$ \\
\hline
\end{tabular}

Fuente: adaptado por los autores de la tabla propuesta por Fan (Fan et al., 2009)

Para esta investigación se asume que el macrofactor se comporta según la distribución de rentabilidades de cada serie bursátil para cada periodo de estudio; además, a diferencia del modelo propuesto por Fan, en vez de definir una probabilidad de imitación igual para el mercado se modela una probabilidad aleatoria $P_{i, j}$ uniformemente distribuida entre $[0,1]$ para cada agente $A_{x, y}$ en el MAC.

En la Figura 2 se evidencia la interfaz gráfica utilizada durante esta investigación; cada pixel representa a un agente $A_{x, y}$, el color de dicho recuadro indica una de las tres posiciones iniciales (comprar, retener y vender), distribuidas aleatoriamente en una grilla de 50x50 unidades dimensionales. La cantidad de iteraciones de cada simulación es equivalente a la cantidad de acciones por año para cada serie bursátil. Una vez determinado el consenso de cada iteración, se estima el coeficiente de Hurst con una ventana temporal que va desde una semana (cinco días) hasta todos los registros del año correspondiente, representando así múltiples horizontes temporales de análisis. Los coeficientes son promediados con el fin de determinar el nivel de memoria o complejidad media de cada serie financiera para dicho periodo. 
Una vez estimado el grado de complejidad de cada serie simulada (a partir de la dinámica de las posiciones finales del mercado), este es contrastado con el grado de complejidad de las series reales (estimado previamente con base en las rentabilidades históricas) con el propósito de determinar si la complejidad de un modelo autómata celular conductual puede representar la complejidad o nivel de memoria del mercado bursátil latinoamericano durante el periodo comprendido entre 2004 y 2016.

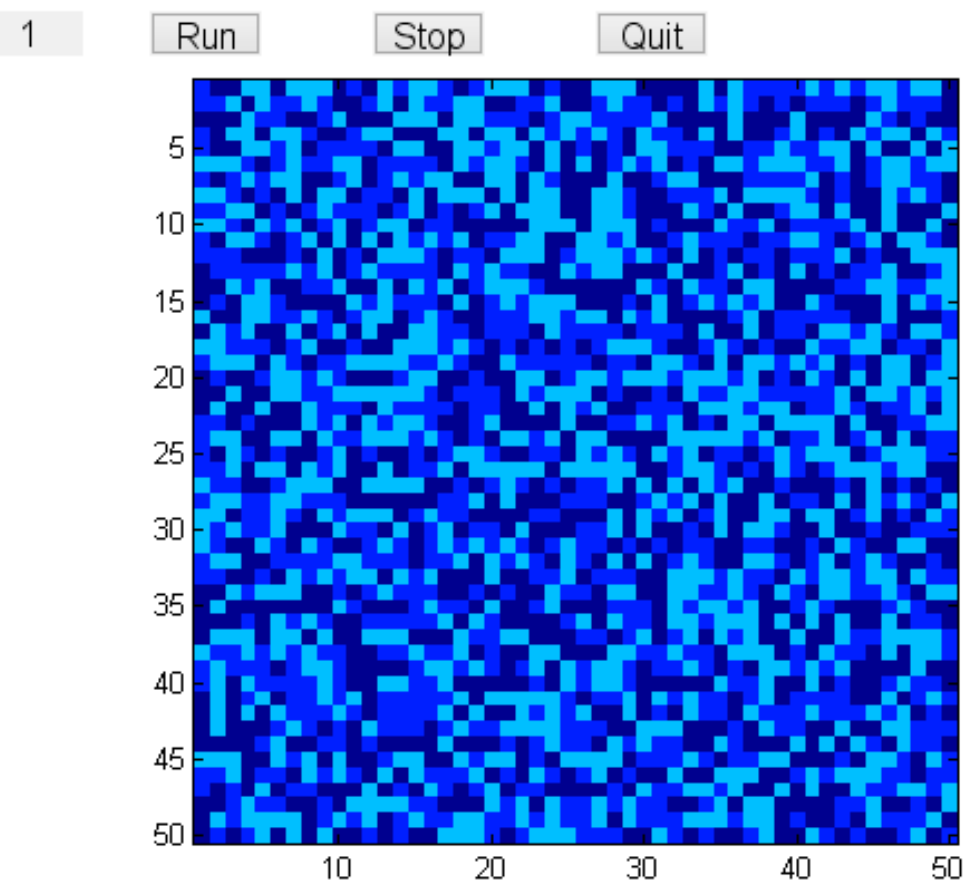

Figura 1. Interfaz gráfica del modelo autómata celular generado en MATLAB

\section{RESULTADOS}

Durante el periodo de estudio, algunos mercados presentaron un aparente patrón de similitud, con cambios marcados en el año 2008 (Figura 2), lo cual puede indicar cierto nivel de correlación; sin embargo, la dinámica económica de ciertos países durante el periodo de análisis presenta una disposición final dispar. Lo anterior no brinda claridad sobre la similitud entre dichas series, sobre todo al tener en cuenta que si bien los indicadores carecen de unidades, se encuentran en magnitudes diferentes. 


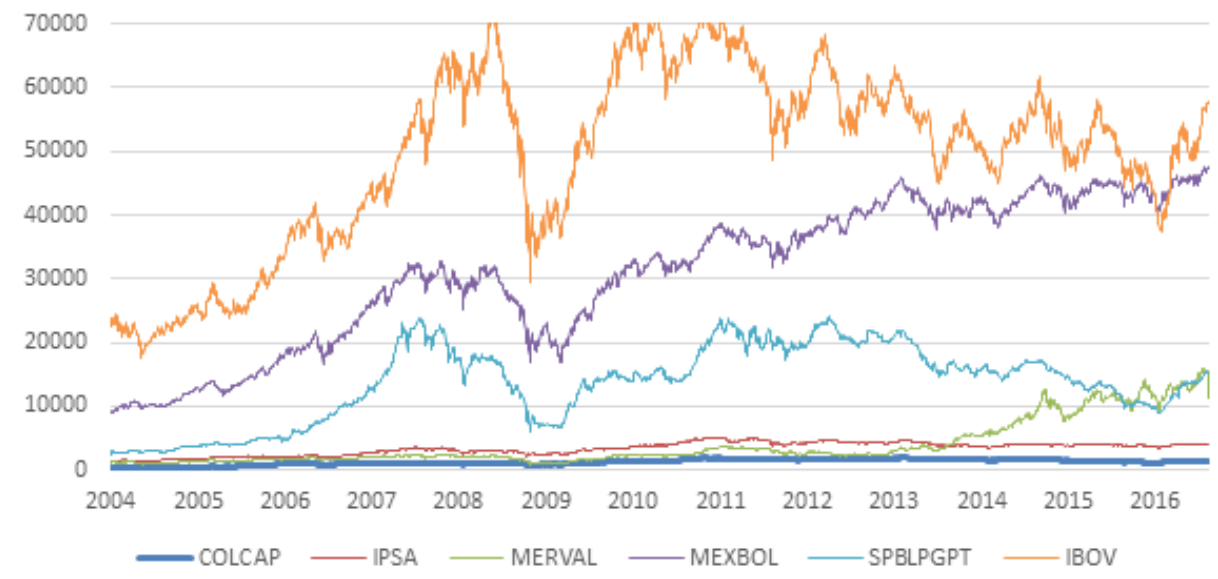

Figura 2. Comportamiento histórico de los principales mercados bursátiles latinoamericanos para el periodo total.

Fuente: los autores, a partir de datos de Bloomberg

Como técnica de análisis de semejanzas, las series financieras son transformadas a una escala común, para ello se evalúa el incremento o decremento porcentual de cada índice respecto al primer dato (correspondiente al 2 de enero de 2004). Una vez transformadas las series, se aprecian con menor "ruido" las similitudes históricas entre los indicadores de cada país (ver Figura 3), y se resalta una tendencia al alza general con una drástica caída entre 2008 y 2009. Entre las características y tendencias de dichas series es de anotar que, una vez superada la crisis de 2008, la economía argentina presentó una veloz recuperación derivada posiblemente del incremento de sus exportaciones a China y Latinoamérica (Miranda, 2014), con una tendencia al alza por encima de los mercados de Colombia y Perú en el periodo poscrisis.

De manera general, se puede inferir que los mercados de Colombia y Chile presentan la mayor estabilidad bursátil, al mostrar un crecimiento medio-alto acompañado de poca variabilidad (coeficientes de variaciones inferiores al $15 \%$ ); seguidos por Brasil, México, Perú y Argentina. No obstante, cabe destacar que Argentina ha mostrado un crecimiento medio similar al de Colombia o Perú, logrando una alta recuperación financiera que se debe, en gran parte, a su "recuperación popular" con la cual logró sobrellevar las crisis de 1999-2001 y 2008-2009 (Aysen, 2015). Debido a dichas características de crecimiento 
(ver Tabla 2) se evidencia una fuerte similitud entre el mercado colombiano, chileno y brasileño.

Por otra parte, para definir el grado de relación lineal histórica entre las series bursátiles, los valores histó- ricos sin transformar de cada índice son contrastados mediante la matriz de correlación registrada en la Tabla 3 y comparados a partir de un ajuste al histograma de frecuencias de sus ren-tabilidades (ver Figura 4).

Tabla 2. Resumen de estadísticas descriptivas para los principales mercados bursátiles latinoamericanos para el periodo total

\begin{tabular}{|c|c|c|c|c|c|c|}
\hline & COLCAP & IPSA & MERVAL & MEXBOL & SPBLPGT & IBOV \\
\hline Media & 1,4781 & 0,7504 & 0,9396 & 1,1675 & 1,5410 & 0,7366 \\
\hline Varianza & 0,2184 & 0,1119 & 0,5550 & 0,2036 & 0,3734 & 0,1123 \\
\hline Desviación estándar & 0,4673 & 0,3345 & 0,7450 & 0,4512 & 0,6110 & 0,3351 \\
\hline Asimetría & $-1,2173$ & $-0,7817$ & 0,7925 & $-0,9841$ & $-1,0594$ & $-1,0386$ \\
\hline Curtosis & 3,8348 & 2,5090 & 2,6080 & 2,8403 & 2,9386 & 3,0911 \\
\hline Mediana & 1,6014 & 0,8910 & 0,7300 & 1,2937 & 1,7550 & 0,8431 \\
\hline Desviación absoluta & 0,3623 & 0,2821 & 0,5954 & 0,3682 & 0,4933 & 0,2672 \\
\hline de la media & & & & & & \\
\hline Moda & 0,7249 & $-0,0156$ & $-0,1429$ & 1,0153 & 1,8245 & $-0,1221$ \\
\hline Mínimo & 0,0000 & $-0,0654$ & $-0,2713$ & 0,0000 & 0,0000 & $-0,2429$ \\
\hline Máximo & 2,0409 & 1,2225 & 2,6759 & 1,6867 & 2,2657 & 1,1865 \\
\hline Rango & 2,0409 & 1,2878 & 2,9472 & 1,6867 & 2,2657 & 1,4294 \\
\hline Cuenta & 2811 & 2811 & 2811 & 2811 & 2811 & 2811 \\
\hline Suma & 4154,9889 & 2109,3757 & 2641,2205 & 3281,7625 & 4331,7679 & 2070,5891 \\
\hline 1er cuartil & 1,3050 & 0,5000 & 0,4081 & 0,8780 & 1,2899 & 0,5586 \\
\hline 3er cuartil & 1,8689 & 0,9948 & 1,1696 & 1,5362 & 1,9785 & 0,9774 \\
\hline Rango intercuartil & 0,5639 & 0,4948 & 0,7615 & 0,6582 & 0,6886 & 0,4188 \\
\hline & & & & & & \\
\hline
\end{tabular}

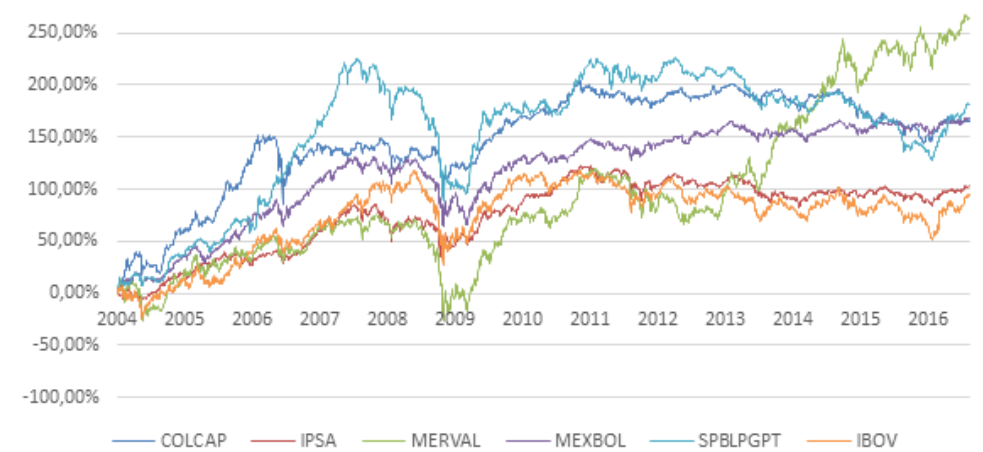

Figura 3. Cambio porcentual en el comportamiento histórico de los principales mercados bursátiles latinoamericanos para el periodo total.

Fuente: los autores a partir de datos de Bloomberg 
Tabla 3. Correlación entre las series financieras sin transformar para el periodo total

\begin{tabular}{|c|c|c|c|c|c|c|}
\hline & COLCAP & IPSA & MERVAL & MEXBOL & SPBLPGPT & IBOV \\
\hline COLCAP & 1 & & & & & \\
\hline IPSA & $\begin{array}{r}0,93793874 \\
9\end{array}$ & 1 & & & & \\
\hline MERVAL & 0,35225621 & $\begin{array}{r}0,43729190 \\
8\end{array}$ & 1 & & & \\
\hline MEXBOL & $\begin{array}{r}0,86347422 \\
3\end{array}$ & $\begin{array}{r}0,89343729 \\
3\end{array}$ & $\begin{array}{r}0,70021209 \\
9\end{array}$ & 1 & & \\
\hline SPBLPGPT & $\begin{array}{r}0,81044020 \\
6\end{array}$ & $\begin{array}{r}0,84910809 \\
2\end{array}$ & $\begin{array}{r}0,12704037 \\
6\end{array}$ & $\begin{array}{r}0,73524561 \\
8\end{array}$ & 1 & \\
\hline IBOV & $\begin{array}{r}0,79741370 \\
7\end{array}$ & $\begin{array}{r}0,84225083 \\
7\end{array}$ & $\begin{array}{r}0,18548332 \\
1\end{array}$ & $\begin{array}{r}0,72135590 \\
5\end{array}$ & $\begin{array}{r}0,87521480 \\
8\end{array}$ & 1 \\
\hline
\end{tabular}

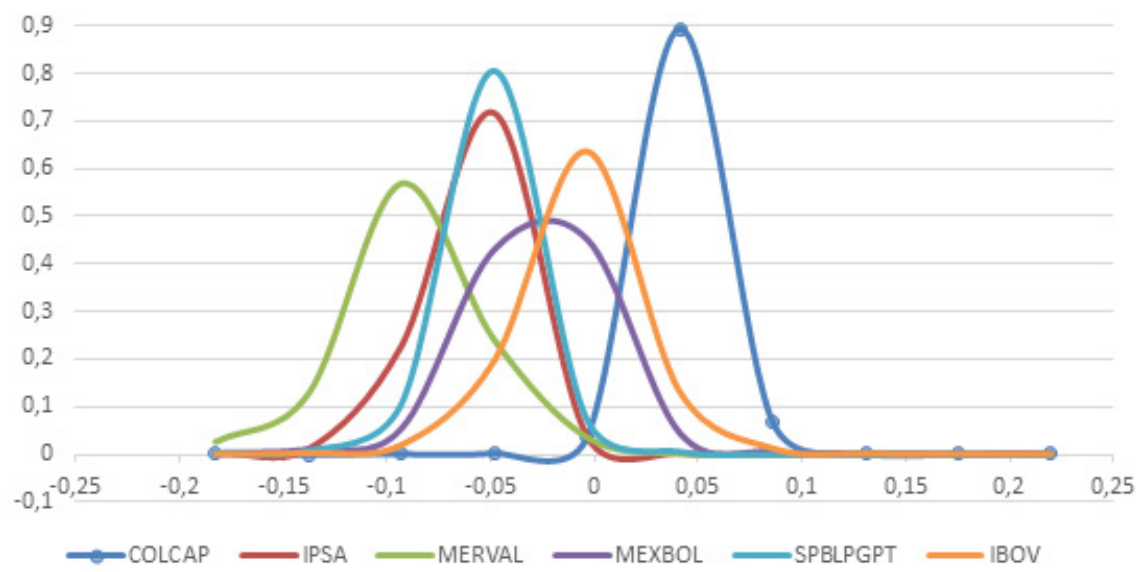

Figura 4. Distribución de rentabilidades de los principales mercados bursátiles latinoamericanos para el periodo total.

Fuente: Los autores a partir de datos de Bloomberg

El mercado bursátil colombiano resalta en Latinoamérica al presentar un incremento medio-alto de sus retornos, acompañado de baja variabilidad; lo cual indica una economía estable en comparación con los otros países bajo estudio; seguido de Brasil, que tradicionalmente se ha considerado como una fuerte economía debido a una sólida estructura productiva, en su mercado se aprecia mayor volatilidad en sus rentabilidades, lo cual podría relacionarse con diversas inestabilidades, entre las que se resaltan las bajas 
exportaciones, la alta devaluación, la inestabilidad sociopolítica y los escándalos de corrupción (Villegas, 2016).

Al analizar el comportamiento histórico de las rentabilidades latinoamericanas (ver Figura 5), se observa que dependiendo del periodo, Colombia, Chile, Argentina, México, Perú y Brasil han presentado comportamientos similares; un claro ejemplo fue antes y durante la crisis de 2008, cuando sus indicadores se mantuvieron al alza y con poca variabilidad; sin embargo, al año siguiente, economías como la peruana fueron fuertemente afectadas con tendencias prolongadas a la baja, mientras que Colombia, Chile y Argentina presentaban un comportamiento volátil.

Cabe resaltar que Brasil, luego del rápido crecimiento en 2005, logró mantener un crecimiento estable aun durante la crisis de 2008, consolidándose a través de su economía productiva; no obstante, a partir de 2011 su economía comenzó a presentar mayor volatilidad, lo cual podría estar relacionado con un estancamiento productivo (Villegas, 2016).

Argentina, en los últimos diez años, ha mostrado una tendencia creciente, acompañada de alta volatilidad, como se observa en los años 2005, 2009 (posterior a la crisis) y 2012 (con problemas en crecimiento, actividad industrial, construcción, inflación y empleo, durante el mandato de Cristina Kirchner) principalmente; lo cual puede exponer una economía creciente pero con alto riesgo. De manera similar, Perú ha evidenciado periodos con tendencia decreciente y gran inestabilidad (como la poscrisis de 2008), que indican una economía con alta volatilidad.

En la Figura 6 se registran los parámetros representativos de las rentabilidades de las principales economías latinoamericanas entre 2004-2016; la conjunción de dichos comportamientos corrobora la tendencia general plasmada en la Figura 4, entendiendo que si bien existen economías con tendencias al alza como es el caso de Argentina, Brasil y México, estas han sido afectadas por diversas crisis sociales, económicas y políticas, aumentando la variabilidad (riesgo) de sus retornos. Así mismo, las economías de Perú y Chile presentan cierta semejanza (alto grado de correlación), empero, han tenido periodos disímiles como en 2009 y 2016, lo cual indica cierta heterogeneidad entre sus comportamientos. 
Apuntes CENES Volumen 36, Número 64

julio - diciembre 2017. Págs. 199-223

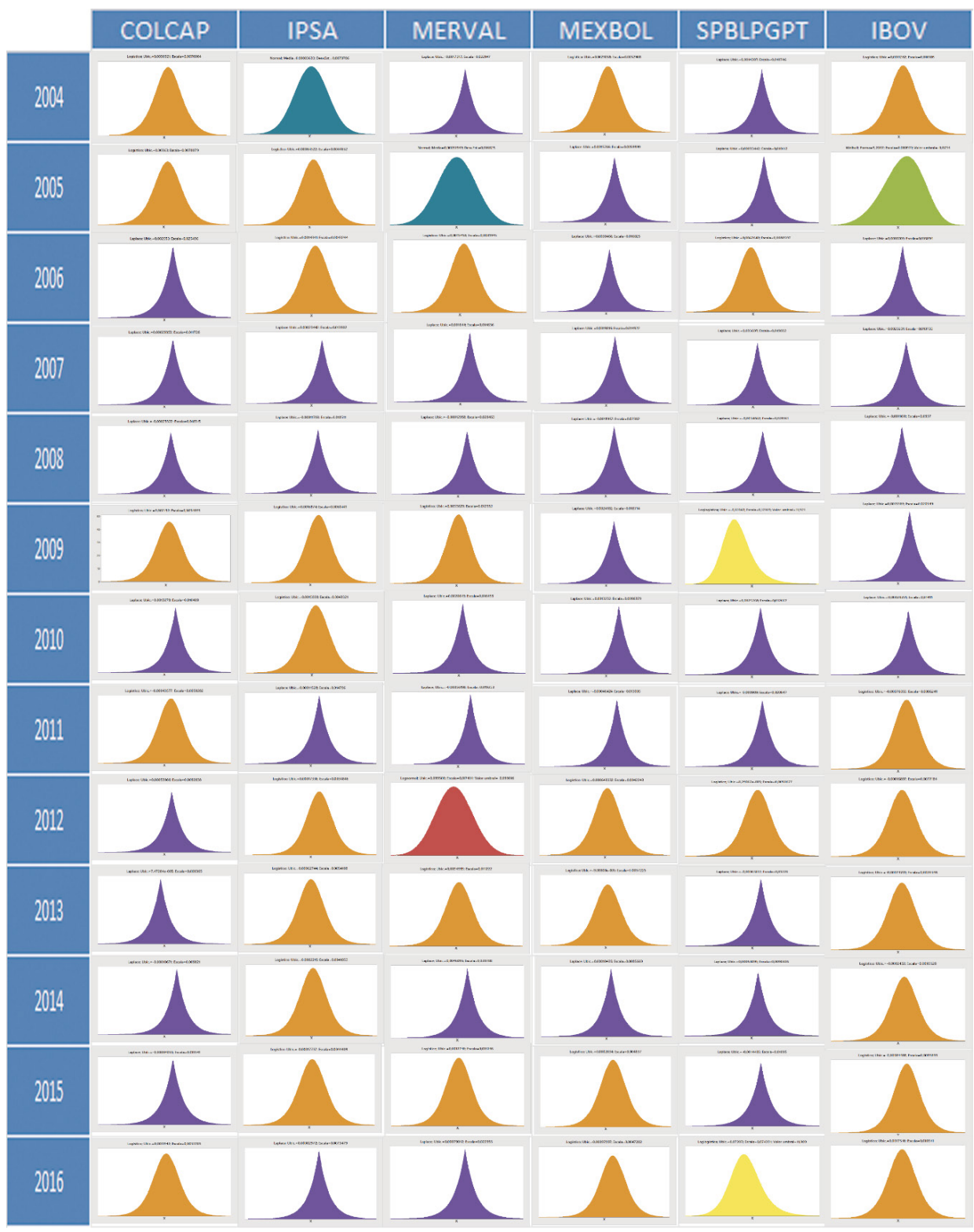

Figura 5. Ajuste de distribución anual de rentabilidades de los principales mercados latinoamericanos entre 2004-2016.

Fuente: los autores, utilizando Minitab 17 
La complejidad del mercado bursátil latinoamericano a partir de un modelo autómata celular conductual

Leonardo Hernán Talero-Sarmiento - Juan Benjamín Duarte-Duarte - Laura Daniela Garcés-Carreño

\begin{tabular}{|c|c|c|c|c|c|c|c|}
\hline & 2004 & 2005 & 2006 & 2007 & 2008 & 2009 & 2010 \\
\hline COLCAP & $\begin{array}{l}0,0030321 \\
0,0076064\end{array}$ & $\begin{array}{c}0,00363 \\
0,0078079\end{array}$ & $\begin{array}{l}0,002553 \\
0,025496\end{array}$ & $\begin{array}{c}0,00029853 \\
0,011726\end{array}$ & $\begin{array}{c}-0,00023322 \\
0,018315\end{array}$ & $\begin{array}{c}0,002132 \\
0,0054893\end{array}$ & $\begin{array}{c}0,0013279 \\
0,010409\end{array}$ \\
\hline IPSA & $\begin{array}{c}0,00083633 \\
0,0078786\end{array}$ & $\begin{array}{c}0,00064522 \\
0,0044652\end{array}$ & $\begin{array}{l}0,0014341 \\
0,0043244\end{array}$ & $\begin{array}{c}0,0023442 \\
0,013902\end{array}$ & $\begin{array}{c}-0,00011703 \\
0,018511\end{array}$ & $\begin{array}{l}0,0016874 \\
0,0060441\end{array}$ & $\begin{array}{l}0,0015038 \\
0,0043321\end{array}$ \\
\hline MERVAL & $\begin{array}{c}0,0017317 \\
0,022147\end{array}$ & $\begin{array}{c}0,00051949 \\
0,016825\end{array}$ & $\begin{array}{l}0,0015458 \\
0,0081945\end{array}$ & $\begin{array}{l}0,001644 \\
0,014636\end{array}$ & $\begin{array}{c}-0,00012958 \\
0,028463\end{array}$ & $\begin{array}{l}0,0016874 \\
0,0060441\end{array}$ & $\begin{array}{c}0,0020643 \\
0,016453\end{array}$ \\
\hline MEXBOL & $\begin{array}{l}0,0021058 \\
0,0052988\end{array}$ & $\begin{array}{l}0,0015766 \\
0,0059999\end{array}$ & $\begin{array}{c}0,0030468 \\
0,016025\end{array}$ & $\begin{array}{c}0,0019816 \\
0,014922\end{array}$ & $\begin{array}{c}-0,0018957 \\
0,02382\end{array}$ & $\begin{array}{c}0,0024182 \\
0,018714\end{array}$ & $\begin{array}{l}0,0013232 \\
0,0098379\end{array}$ \\
\hline SPBLPGPT & $\begin{array}{c}0,0014387 \\
0,010746\end{array}$ & $\begin{array}{c}0,00093442 \\
0,010112\end{array}$ & $\begin{array}{l}0,0042648 \\
0,0088597\end{array}$ & $\begin{array}{c}0,0033607 \\
0,019032\end{array}$ & $\begin{array}{c}-0,0034842 \\
0,028161\end{array}$ & $\begin{array}{c}-0,12042 \\
.0,12169 \\
11,921\end{array}$ & $\begin{array}{c}0,0025268 \\
0,012667\end{array}$ \\
\hline IBOV & $\begin{array}{c}0,0011262 \\
0,010105\end{array}$ & $\begin{array}{c}5,2067 \\
0,080971 \\
-0,0734\end{array}$ & $\begin{array}{c}0,0010703 \\
0,016894\end{array}$ & $\begin{array}{c}0,0023231 \\
0,018733\end{array}$ & $\begin{array}{c}-0,0011611 \\
0,0337\end{array}$ & $\begin{array}{c}0,0013365 \\
0,020549\end{array}$ & $\begin{array}{c}0,00029251 \\
0,014111\end{array}$ \\
\hline
\end{tabular}

\begin{tabular}{|c|c|c|c|c|c|c|c|}
\cline { 2 - 8 } & $\mathbf{2 0 1 1}$ & $\mathbf{2 0 1 2}$ & $\mathbf{2 0 1 3}$ & $\mathbf{2 0 1 4}$ & $\mathbf{2 0 1 5}$ & $\mathbf{2 0 1 6}$ & CONVENCIONES \\
\hline COLCAP & $-0,00043577$ & 0,00053986 & 0,0000747284 & $-0,00010671$ & $-0,00084053$ & 0,0011924 & $\begin{array}{c}\text { Logistic } \\
\text { Media: } \\
\text { Desviación: }\end{array}$ \\
\hline IPSA & 0,0059282 & 0,0092838 & 0,008365 & 0,009821 & 0,011541 & 0,0053705 & $\begin{array}{c}\text { Normal } \\
\text { Media: }\end{array}$ \\
\hline MERVAL & 0,00011528 & 0,00017336 & $-0,00062744$ & 0,0002219 & $-0,00017737$ & 0,00082972 \\
Desviación:
\end{tabular}

Figura 6. Parámetros descriptivos para los ajuste de distribución anual de rentabilidades de los principales mercados latinoamericanos entre 2004-2016.

Fuente: los autores, utilizando@Risk 7.5

Otra manera de contrastar el comportamiento económico latinoamericano es a partir de su complejidad, estimada en este estudio mediante el coeficiente de Hurst. Al evaluar bajo esa metodología la economía latinoamérica año a año, se encuentra que los países que han presentado una mayor volatilidad 
y dispersión con funciones de comportamiento normal, poseen un nivel de memoria menos persistente (más cercano a 0.5$)$ y por tanto más eficiente, lo cual coincide en algunos periodos con lo expuesto por Duarte y Mascareñas (2014b), quienes aplican el test Jarque-Bera, la prueba de bondad de ajuste de la chi-cuadrado y tres versiones de la caminata aleatoria (RW) a diversas series latinoamericanas.

Si bien existen ligeras diferencias en la memoria de los índices bajo estudio (series complejas con tendencia persistente), como se aprecia en la Figura 7, los mercados latinoamericanos son muy similares en su comportamiento, tendencias y memorias. De hecho, posiblemente, estos disten aún de presentar un comportamiento completamente eficiente, lo que puede implicar menor variabilidad $\mathrm{y}$, por ende, evidenciar tendencias en sus retornos menos normales y más leptocurticos como el caso de las funciones Lognormal, Logistic y Laplace.

Las estimaciones del coe iciente de Hurst para las series reales (Tabla 4) di ieren sustancialmente de las estimaciones obtenidas mediante la simulación del mercado bursátil arti icial con agentes conductuales (Tabla 5), puesto que estas últimas muestran un comportamiento antipersistente, que indica una aparente relación entre los tipos de distribución y el intervalo de con ianza, siendo ligeramente más pequeños aquellos derivados de funciones más leptocúrticas.

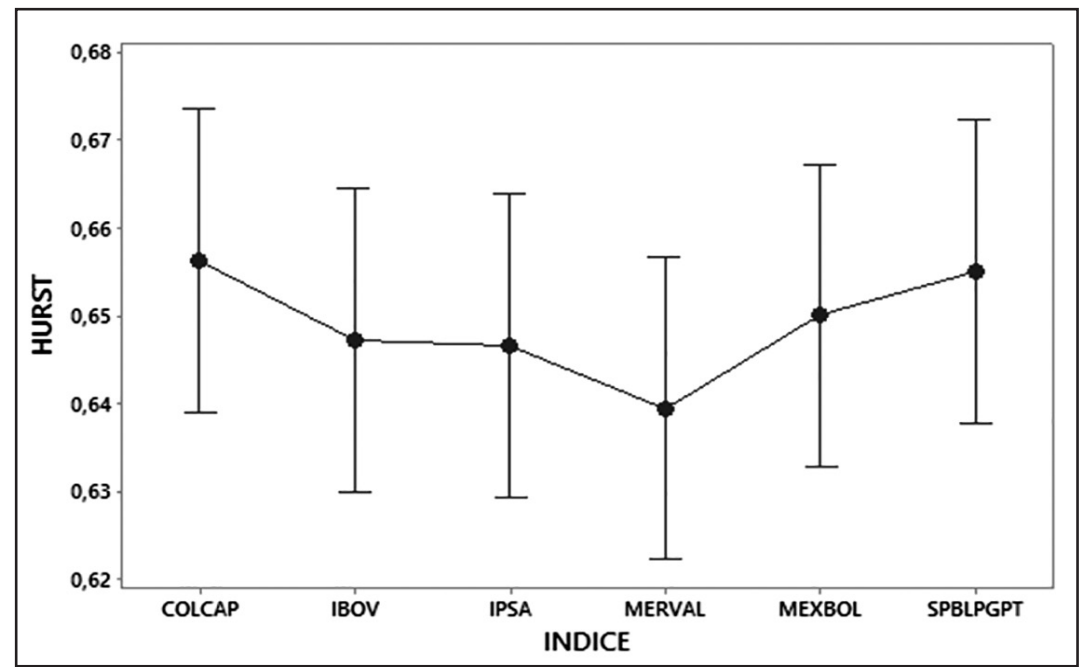

Figura 7. Intervalos de confianza con $95 \%$ de confianza del coeficiente de Hurst estimado anualmente entre 2004-2016 para los principales mercados bursátiles latinoamericanos.

Fuente: los autores, utilizando Minitab 17 
La complejidad del mercado bursátil latinoamericano a partir de un modelo autómata celular conductual Leonardo Hernán Talero-Sarmiento - Juan Benjamín Duarte-Duarte - Laura Daniela Garcés-Carreño

Tabla 4. Estimación del coeficiente de Hurst entre 2004-2016 para los principales mercados bursátiles de Latinoamérica

\begin{tabular}{|r|r|r|r|r|r|r|}
\hline & COLCAP & IPSA & MERVAL & MEXBOL & SPBLPFP & \multicolumn{1}{|l|}{ IBOV } \\
\hline 2004 & 0,6736 & 0,6519 & 0,638 & 0,6644 & 0,6242 & 0,6427 \\
\hline 2005 & 0,646 & 0,6826 & 0,6619 & 0,6758 & 0,6731 & 0,6735 \\
\hline 2006 & 0,6158 & 0,6537 & 0,6597 & 0,6116 & 0,6686 & 0,635 \\
\hline 2007 & 0,6164 & 0,6496 & 0,6134 & 0,6568 & 0,6757 & 0,6513 \\
\hline 2008 & 0,6366 & 0,6392 & 0,6476 & 0,6457 & 0,667 & 0,6436 \\
\hline 2009 & 0,6391 & 0,6496 & 0,6451 & 0,6535 & 0,6537 & 0,6406 \\
\hline 2010 & 0,8203 & 0,5673 & 0,6273 & 0,6719 & 0,6681 & 0,6567 \\
\hline 2011 & 0,6338 & 0,6708 & 0,6552 & 0,6358 & 0,645 & 0,6485 \\
\hline 2012 & 0,6678 & 0,6748 & 0,6485 & 0,6466 & 0,6712 & 6707 \\
\hline 2013 & 0,6718 & 0,6603 & 0,6338 & 0,6599 & 0,6565 & 0,659 \\
\hline 2014 & 0,6682 & 0,6552 & 0,6303 & 0,6599 & 0,663 & 0,6692 \\
\hline 2015 & 0,6415 & 0,6559 & 0,6559 & 0,6723 & 0,6483 & 0,638 \\
\hline 2016 & 0,6017 & 0,5946 & 0,5962 & 0,5962 & 0,6021 & 0,585 \\
\hline
\end{tabular}

Tabla 5. Estimación del coeficiente de Hurst entre 2004-2016 mediante la simulación de un modelo autómata celular con macrofactor probabilístico latinoamericano y capacidad de imitación individual

\begin{tabular}{|r|r|r|r|r|r|r|}
\hline & COLCAP & \multicolumn{1}{|c|}{ IPSA } & MERVAL & MEXBOL & \multicolumn{1}{|c|}{ SPBLPFT } & \multicolumn{1}{|c|}{ IBOV } \\
\hline 2004 & 0,4204 & 0,4121 & 0,5079 & 0,5249 & 0,4788 & 0,4892 \\
\hline 2005 & 0,5139 & 0,4242 & 0,557 & 0,5395 & 0,5058 & 0,4759 \\
\hline 2006 & 0,4677 & 0,4496 & 0,4368 & 0,5053 & 0,3964 & 0,4621 \\
\hline 2007 & 0,5192 & 0,4912 & 0,5282 & 0,4573 & 0,4823 & 0,4814 \\
\hline 2008 & 0,5395 & 0,4625 & 0,4556 & 0,4712 & 0,4537 & 0,4462 \\
\hline 2009 & 0,4327 & 0,5249 & 0,4636 & 0,5361 & 0,407 & 0,5272 \\
\hline 2010 & 0,4781 & 0,4916 & 0,5027 & 0,4777 & 0,4628 & 0,4862 \\
\hline 2011 & 0,4538 & 0,5343 & 0,4543 & 0,4189 & 0,5354 & 0,4264 \\
\hline 2012 & 0,4586 & 0,4481 & 0,4405 & 0,4163 & 0,4872 & 0,4684 \\
\hline 2013 & 0,5334 & 0,4842 & 0,492 & 0,4939 & 0,4675 & 0,4123 \\
\hline 2014 & 0,5163 & 0,4864 & 0,4843 & 0,4155 & 0,526 & 0,4377 \\
\hline 2015 & 0,5055 & 0,4623 & 0,5232 & 0,4367 & 0,4559 & 0,5615 \\
\hline 2016 & 0,3869 & 0,4906 & 0,4459 & 0,3476 & 0,3603 & 0,3938 \\
\hline
\end{tabular}




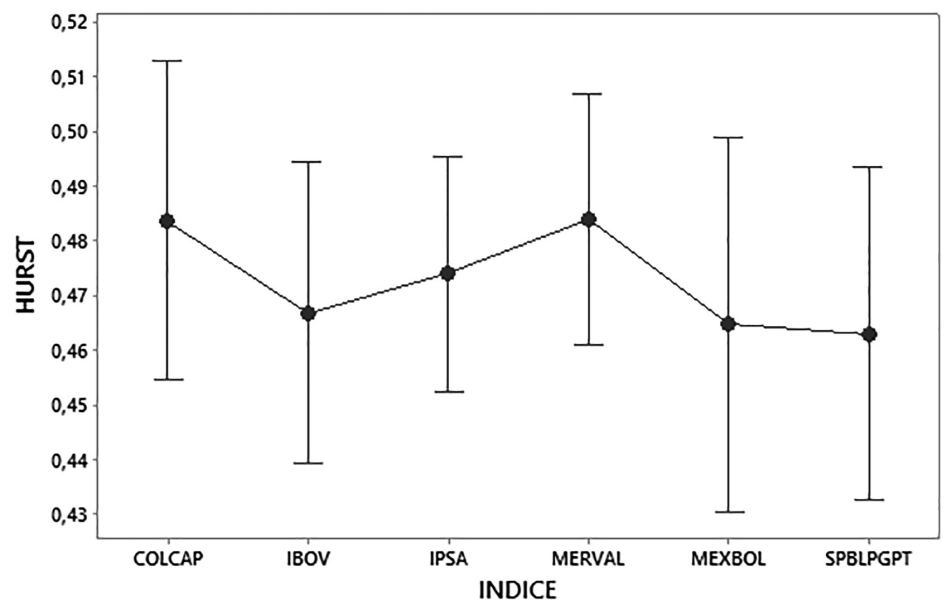

Figura 8. Intervalos de confianza con $95 \%$ de confianza del coeficiente de Hurst simulado anualmente entre 2004-2016 para los principales mercados bursátiles latinoamericanos.

Fuente: Los autores utilizando Minitab 17

Una vez contrastados los niveles de memoria/complejidad de las series reales y simuladas, se determina que, en general, no existe diferencia entre el comportamiento medio de los índices bursátiles; ahora bien, dicha similitud puede presentar variaciones dependiendo del periodo de estudio (año) y evidentemente del origen de la serie por estudiar (real, simulada). El comportamiento de los residuales se evidencia en la Figura 9.

Tabla 6. Análisis de varianza para los coeficientes de Hurst estimados.

Fuente: los autores, a partir de Minitab 17

\begin{tabular}{|l|r|r|r|r|r|r|}
\hline Fuente & GL & & SC Ajust. & MC Ajust. & Valor F & Valor P \\
\hline IIndice & 5 & 0,00048 & 0,0001 & 0,06 & 0,997 \\
\hline $\begin{array}{l}\text { Origen de } \\
\text { datos }\end{array}$ & 1 & 1,17671 & 1,17671 & 743,84 & 0 \\
\hline Año & 12 & 0,06274 & 0,00523 & 3,31 & 0 \\
\hline Error & 137 & 0,21673 & 0,00158 & & \\
\hline $\begin{array}{l}\text { Falta de } \\
\text { ajustes }\end{array}$ & 136 & 0,18467 & 0,00136 & 0,04 & 1 \\
\hline Error Puro & 1 & 0,03206 & 0,03206 & & \\
\hline Total & 155 & 1,4559 & & & \\
\hline
\end{tabular}




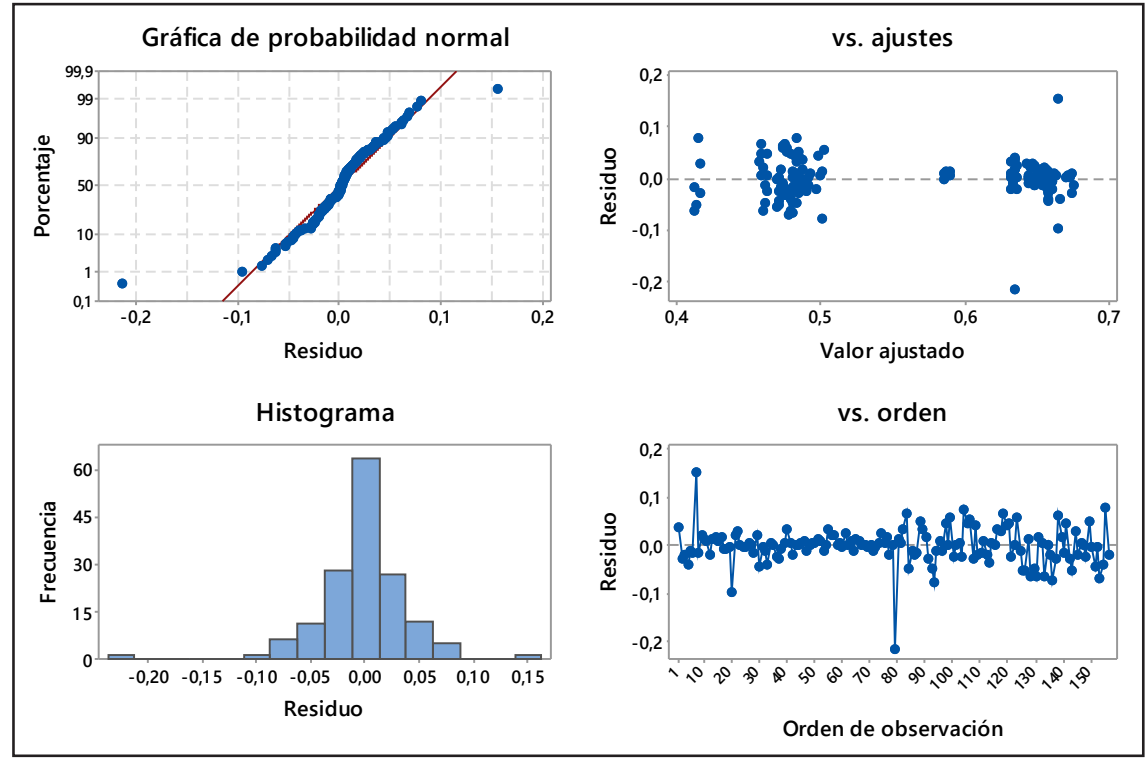

Figura 9. Gráficas de residuos de Hurst.

Fuente: los autores, utilizando Minitab 17

\section{DISCUSIÓN}

Latinoamérica se ha destacado por tener ciertas características afines en sus economías, derivadas posiblemente de similitudes sociopolíticas (entre otros aspectos); diversos estudios se han realizado con el fin de determinar si sus principales representantes (Brasil, México, Colombia, Perú, Chile y Argentina) son eficientes y cuáles son los factores que influyen en dicho comportamiento. El problema radica en que dependiendo del método y el periodo, los investigadores pueden encontrar tendencias diferentes, las cuales pueden ser catalogadas en la aceptación de la hipótesis de mercado eficiente, rechazo de la hipóte- sis de mercado eficiente y mejora en la eficiencia (Duarte \& Mascareñas, 2014a).

Debido a lo anterior, proponer una estructura sistemática del comportamiento de los retornos (incluyendo el ajuste probabilístico, parámetros de distribución y periodo evaluado) facilita el contraste con otros estudios y da indicios visuales sobre el comportamiento de los principales mercados latinoamericanos, permitiendo contrastar entre sí y a lo largo del tiempo la evolución de los mismos.

Por otra parte, las economías de Colombia, Chile, Argentina, México, Perú y Brasil, a pesar de haber 
presentado evoluciones diferentes a lo largo del tiempo, tienen marcadas tendencias en común, lo cual se evidencia en el comportamiento de sus rentabilidades (ver Figura 5). Estos se caracterizan por ser mercados al alza con alta volatilidad, lo cual puede estar relacionado con las crisis sociales, económicas y políticas presentadas en esos años, ya que en el mercado bursátil se evidencian anomalías alrededor de los periodos de crisis (Duarte, Garcés \& Sierra, 2016).

Si bien durante el estudio se estimó el nivel de memoria y en contraparte la eficiencia de los mercados, cabe resaltar que existen múltiples formas de hallar el coeficiente de Hurst y que este, al ser una estimación sensible a la presencia de memoria a corto plazo, puede variar según la metodología; sin embargo, al contrastar el comportamiento real de las series históricas con el nivel de memoria de los mercados bursátiles artificiales adaptados del modelo creado por Fan (2009), es posible identificar que una bolsa simulada con agentes conductuales en vez de racionales, no parece representar el nivel de complejidad latinoamericano, al menos con los parámetros de macrofactor e imitación, lo cual se relacionaría con el efecto manada, y este es solo uno de los tantos sesgos posibles.

Otro elemento para considerar es la fuente de los datos; durante este estudio se utilizaron los precios de cierre de cada acción obtenidos mediante Bloomberg; sin embargo, existen discrepancias entre la información disponible para cada índice. Debido a lo anterior y teniendo en cuenta que el objetivo primario de esta investigación se relaciona con la comparación entre mercados, fue necesario depurar el data set para poder comparar históricamente las series, lo cual puede distorsionar ligeramente los resultados.

Finalmente, para futuras investigaciones es recomendable trabajar con modelos autómatas celulares generadores de precios, a fin de realizar contrastes más profundos entre las rentabilidades simuladas y los históricos de las series financieras, además, con el propósito de considerar la autodependencia en el tiempo, hacer los análisis del coeficiente de Hurst con la variante propuesta por Andrew Lo (1991).

\section{CONCLUSIÓN}

Debido a la configuración de sus mercados bursátiles, durante el periodo comprendido entre 2004 a 2016, la economía latinoamérica presentó una baja eficiencia con comportamientos leptocúrticos en el retorno de sus activos, indicando cierto grado de memoria (persistencia en las series), el cual se evidencia en la rapidez con que esta entró y salió de crisis, determinando que a la luz de los resultados no es posible aceptar la hipótesis propuesta por Fama (1970), ya que no se 
verifica el comportamiento de caminatas aleatorias.

Teniendo en cuenta lo anterior, se concluye que el modelo conductual propuesto durante esta investigación no logra explicar el comportamiento económico latinoamericano y si bien las series artificiales distaron de indicar eficiencia, mostraron una tendencia caótica (antipersistente), por tal razón, es necesario a partir de futuras investigaciones determinar qué otros elementos económicos y tipos de agentes es necesario modelar, para conocer, describir y, de ser posible, predecir el comportamiento económico latinoamericano mediante la simulación. 
Apuntes CENES Volumen 36, Número 64

julio - diciembre 2017. Págs. 199-223

\section{REFERENCIAS}

Atman, A. P. F. \& Gonçalves, B. A. (2012). Influence of the Investor's Behavior on the Complexity of the Stock Market. Brazilian Journal of Physics, 42(1-2), 137-145. https://doi.org/10.1007/s13538-011-0057-7

Aysen, M. (2015). Argentina y su desarrollo posterior a la crisis financiera. Problemas del Desarrollo, 46(180), 151-174. Recuperado de http://doi. org/10.1016/S0301-7036(15)72123-1

Bakker, L., Hare, W., Khosravi, H. \& Ramadanovic, B. (2010). A Social Network Model of Investment Behaviour in the Stock Market. Physica A: Statistical Mechanics and its Applications, 389(6), 1223-1229. Retrieved from http:// doi.org/10.1016/j.physa.2009.11.013

Bartolozzi, M. \& Thomas, A. W. (2005). Stochastic Cellular Automata Model for Stock Market Dynamics. Physical Review, 73, 1-17.

Blasco de Las Heras, N. \& Santamaría, R. (1994). Memoria a largo plazo en el mercado de valores español: una aproximación a través del análisis $\mathrm{R} / \mathrm{S}$. Investigaciones Económicas, 28(3), 571-583. Recuperado de http://ftp. fundacionsepi.es/investigacion/revistas/paperArchive/Sep1994/v18i3a9. pdf

Duarte, J. B., Garcés, L. D. \& Sierra, K. J. (2016). Efecto manada en sectores económicos de las bolsas latinoamericanas: una visión pre y poscrisis subprime. Contaduría y Administración, 61(2), 298-323. Recuperado de http:// doi.org/10.1016/j.cya.2015.12.002

Duarte, J. B. \& Mascareñas, J. M. (2014a). ¿ Han sido los mercados bursátiles eficientes informacionalmente? Apuntes del Cenes, 33(57), 117-146.

Duarte, J. B. \& Mascareñas, J. M. (2014b). Comprobación de la eficiencia débil en los principales mercados financieros latinoamericanos. Estudios Gerenciales, 30(133), 365-375. Recuperado de http://doi.org/10.1016/j.estger.2014.05.005

Fama, E. (1970, May). Efficient Captial Markets: A Review of Theory and Empirical Work. The Journal of Finance, 25(2). Retrieved from http://doi. org/10.1111/j.1540-6261.1970.tb00518.x 
Fan, Y., Ying, S.-J., Wang, B.-H. \& Wei, Y.-M. (2009). The Effect of Investor Psychology on the Complexity of Stock Market: An Analysis Based on Cellular Automaton Model. Computers \& Industrial Engineering, 56(1), 63-69. Retrieved from http://doi.org/10.1016/j.cie.2008.03.015

Lo, A.W. (1991). Long-Term Memory in Stock Market Prices. Econometrica, 59(5), 1279. Retrieved from http://www.jstor.org/stable/2938368?origin $=$ crossref

Lo, A. W. (2005). Reconciling Efficient Markets with Behavioral Finance: The Adaptive Markets Hypothesis. Journal of Investment Consulting, 920(617).

Malkiel, B. G. \& Fama, E. F. (1970). Efficient Capital Markets: A Review of Theory and Empirical Work. The Journal of Finance, 25(2), 383-417. Retrieved from http://doi.org/10.1111/j.1540-6261.1970.tb00518.x

Mandelbrot, B. (1972). Statistical Methodology for Nonperiodic Cycles: From the Covariance to R/S Analysis. Annals of Economic and Social Measurement, 1(3), 259-290.

Miranda, R. (2014). Comercio y política: Argentina entre las potencias y las no potencias. Latinoamérica, Revista de Estudios Latinoamericanos, 59, 4167. Recuperado de http://doi.org/10.1016/S1665-8574(14)71725-9

Mozafari, M. \& Alizadeh, R. (2013). A Cellular Learning Automata Model of Investment Behavior in the Stock Market. Neurocomputing, 122, 470-479. Retrieved from http://doi.org/10.1016/j.neucom.2013.06.002

Qiu, G., Kandhai, D. \& Sloot, P. M. A. (2007). Understanding the Complex Dynamics of Stock Markets Through Cellular Automata. Physical Review, 75(4), 46116-11. Retrieved from http://doi.org/10.1103/PhysRevE.75.046116

Shiller, R. J. (2003). From Efficient Markets Theory to Behavioral Finance. Journal of Economi Perspectives, 17(1055), 83-104.

Sierra, G. (2007). Procesos de Hurst y movimiento browniano fraccional en mercados fractales: Valuación y aplicaciones a los derivados y Finanzas. Monterrey: Instituto Tecnológico de Estudios Superiores de Monterrey. 
Stefan, F. M. \& Atman, A. P. F. (2015). Is there Any Connection Between the Network Morphology and the Fluctuations of the Stock Market Index? Physica A: Statistical Mechanics and its Applications, 419, 630-641. Retrieved from http://doi.org/10.1016/j.physa.2014.10.026

Villegas, P. H. L. (2016). Latinoamérica ante las transformaciones del orden monetario y financiero mundial. Economía Informa, 396, 67-83. Recuperado de http://doi.org/10.1016/j.ecin.2016.01.004

Wei, Y., Ying, S., Fan, Y. \& Wang, B.-H. (2003). The Cellular Automaton Model of Investment Behavior in the Stock Market. Physica A: Statistical Mechanics and its Applications, 325(3), 507-516. Retrieved from http://doi. org/10.1016/S0378-4371(03)00144-4

Ying, S. \& Fan, Y. (2014). Complexity in the Chinese Stock Market and its Relationships with Monetary Policy Intensity. Physica A, 394, 338-345. Retrieved from http://doi.org/10.1016/j.physa.2013.09.047

Zhou, T., Zhou, P.-L., Wang, B.-H., Tang, Z.-N. \& Liu, J. (2004). Modeling Stock Market Based on Genetic Cellular Automata. Science And Technology, 18, 2697-2702. Retrieved from http://doi.org/10.1142/S0217979204025932 
